www.mdpi.com/journal/applsci

Article

\title{
Ab Initio Study of Lattice Site Occupancies in Binary Sigma Phases Using a Single-Site Mean Field Model
}

\author{
Evgeniya Kabliman ${ }^{1}{ }^{*}$, Andrei V. Ruban ${ }^{2}$, Peter Blaha ${ }^{1}$, Oleg Peil ${ }^{3}$ and Karlheinz Schwarz ${ }^{1}$ \\ ${ }^{1}$ Institute of Materials Chemistry, Vienna University of Technology, Getreidemarkt 9/165-TC, A-1060 \\ Vienna, Austria \\ ${ }^{2}$ Applied Material Physics, Department of Materials Science and Engineering, Royal Institute of \\ Technology, Brinellvägen 23, SE-100 44 Stockholm, Sweden \\ ${ }^{3}$ I. Institut of Theoretical Physics, Hamburg University, Jungiusstrasse 9, 20355 Hamburg, Germany \\ * Author to whom correspondence should be addressed; E-Mail: evgeniya @ theochem.tuwien.ac.at; \\ Tel.: +43-1-58801-15671; Fax: +43-1-58801-15698.
}

Received: 3 July 2012; in revised form: 7 August 2012 / Accepted: 13 August 2012 /

Published: 3 September 2012

\begin{abstract}
The site occupation of binary Fe-Cr, Co-Cr, Re-W and Fe-V sigma phases is studied in the present work with a first-principles-based single-site mean field theory. We show that the alloy components in these systems exhibit similar site preferences except for the Re-W system, where the occupation of two sites is reversed in agreement with previously published works. In case of the $\mathrm{FeV}$ sigma phase, for which the size mismatch between the alloy components is large, we also include into our consideration the effect of local lattice relaxations. The obtained results are found in good agreement with the experimental data and previous theoretical studies.
\end{abstract}

Keywords: sigma phase; site occupancies; binary systems; first principles

\section{Introduction}

In 1923 the $\sigma$-phase was first observed by Bain as a hard non-magnetic intermediate phase which was formed during a long-time high-temperature annealing in the $\mathrm{Fe}-\mathrm{Cr}$ alloy with a nearly equiatomic composition. Being extremely brittle, the $\sigma$-phase substantially degrades the mechanical properties of different types of alloys, mainly stainless steels, Fe- and Ni-based superalloys. The precipitation of 
this phase decreases ductility and corrosion resistance, while it increases brittleness and promotes the formation of cracks leading to high temperature failure, metal dusting and loss of toughness during heat treatment or welding [1].

The $\sigma$-phase has a topologically closed packed structure, which is defined by a tetragonal unit cell (space group $P 4_{2} / \mathrm{mnm}$ ) containing 30 atoms. These atoms are located in five nonequivalent groups of sites or sublattices $(\mathrm{A}, \mathrm{B}, \mathrm{C}, \mathrm{D}, \mathrm{E})[2,3]$. One often uses the following formula $A_{2}^{12} B_{4}^{15} C_{8}^{14} D_{8}^{12} E_{8}^{14}$ to describe the $\sigma$-phase structure, where bottom and top indices denote the site multiplicities and coordination numbers for each sublattice, respectively. The crystal structure of the $\sigma$-phase can be determined as a stacking of the kagomé tiles with the $\mathrm{A}(0,0,0), \mathrm{B}(x, x, 0), \mathrm{C}(x, y, 0)$ and $\mathrm{D}(x, y, 0)$ atoms lying in the tile plane and the $\mathrm{E}(x, x, z)$ atoms placed between the pseudo-hexagonal rings of kagomé tile [3] as shown in Figure 1.

Figure 1. The tetragonal unit cell of the $\sigma$-phase structure with 5 crystallographically inequivalent sites (A, B, C, D, E).
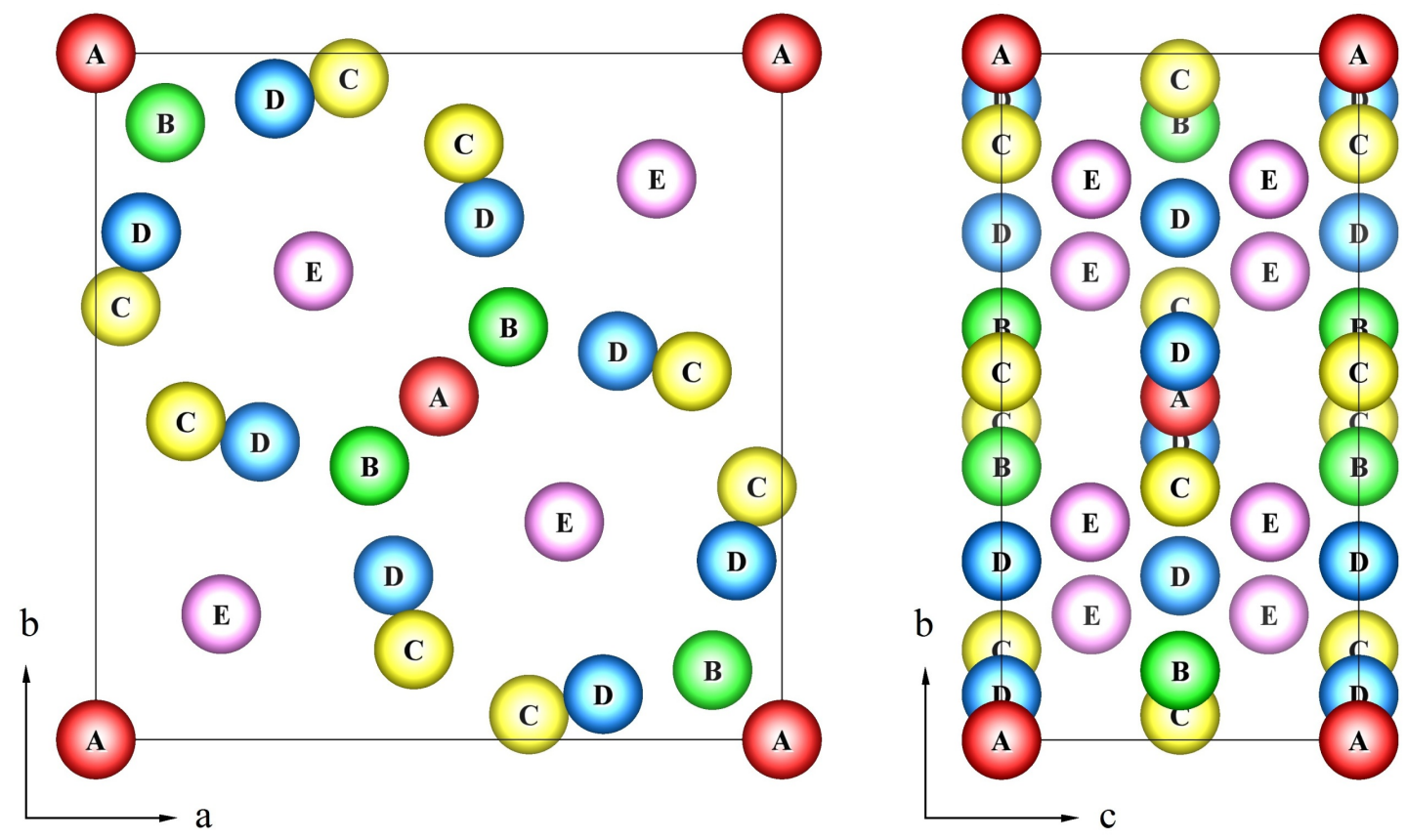

It is known that the nonequivalent $\sigma$-phase sites are partially occupied by all the alloy components, but with certain preferences. Already in 1955 using neutron and X-ray diffraction studies in Fe-V, Ni-V and Mn-Cr alloys Kasper and Waterstrat suggested a general scheme for the ordering in binary $\sigma$-phases of first row transition metal elements and those containing Mo [4]. They found that the highly coordinated $\mathrm{B}$ sites are predominately occupied by the elements to the left of $\mathrm{Mn}$ in the periodic table ( $\mathrm{V}, \mathrm{Cr}, \mathrm{Mo}$ ), whereas the $\mathrm{A}$ and $\mathrm{D}$ sublattice with an icosahedral environment are almost exclusively occupied by elements to the right of manganese ( $\mathrm{Fe}, \mathrm{Co}, \mathrm{Ni}$ ). In contrast, the $\mathrm{C}$ and $\mathrm{E}$ sites are partially occupied by both components and the proportions depend on the alloy composition and external conditions. Although some preferences in the site occupation could be estimated for some binary $\sigma$-phases, the knowledge of the atomic site distribution in a wide temperature and composition range is still missing. Such information is useful for studying the $\sigma$-phase stability and its precipitation. However, it is quite 
hard to obtain these data experimentally due to sluggish kinetics of the transformation, difficulties in getting high purity samples and satisfying some other technological requirements.

In this context, an accurate theoretical study based on state-of-the-art first-principles methods combined with a proper statistical model can be used in order to determine the site preferences of the alloy components in the $\sigma$-phase. Recently the site occupation in the $\sigma$-phase has been widely studied by such methods [5-13]. However, most of these methods were based on a formalism, which is quite computationally demanding. Alternatively, we have recently proposed a simpler approach to calculate the site preference in the binary $\sigma$-phases [14-16]. It is based on a simple single-site mean-field model, which recently has demonstrated to be able to produce quite accurate results for the site occupancy of $\mathrm{Fe}$ and $\mathrm{Cr}$ in the $\mathrm{Fe}-\mathrm{Cr} \sigma$-phase. In this paper we briefly describe this method and apply it to study the distribution of alloy components in other binary $\sigma$-phase, namely, $\mathrm{Co}-\mathrm{Cr}$, Re-W and $\mathrm{Fe}-\mathrm{V}$, for which experimental and theoretical information is available.

\section{Single-Site Model for the Free Energy}

In order to find the atomic occupancies of the $\sigma$-phase sublattices, we minimize the Helmholtz free energy $F$, which can be written in the following form for a given composition and temperature [14-16]:

$$
F\left(\left\{c_{\alpha}\right\}\right)=E_{\text {tot }}\left(\left\{c_{\alpha}\right\}\right)-T\left(S_{\text {conf }}+S_{\text {magn }}\right)
$$

Here $E_{t o t}\left(\left\{c_{\alpha}\right\}\right)$ is the total energy of the $\sigma$-phase, $c_{\alpha}$ the concentration of the reference component (e.g., $\mathrm{Cr}$ in the Fe-Cr alloy), and $\alpha$ is the $\mathrm{A}, \mathrm{B}, \mathrm{C}, \mathrm{D}$, or E site. We assume here that the alloy components are distributed randomly on each site and there is no mutual correlation between them on different sublattices. As has been demonstrated in [16], this is a valid approximation at high temperatures at least in the case of the Fe-Cr $\sigma$-phase. Thus, the total energy (per unit cell), $E_{t o t}\left(\left\{c_{\alpha}\right\}\right)$, can be written in a simplified form as

$$
E_{t o t}\left(\left\{c_{\alpha}\right\}\right)=E_{0}(c)+\sum_{\alpha=1,4(A, D)} n_{\alpha} J_{\alpha}^{(1)} c_{\alpha}
$$

where $n_{\alpha}$ is the number of sites at sublattice $\alpha$, and $J_{\alpha}^{(1)}$ are the on-site interaction parameters, defined as

$$
J_{\alpha}^{(1)}=\left.\left[\partial E_{t o t} / \partial c_{\alpha}-\partial E_{t o t} / \partial c_{E}\right]\right|_{c_{a l l o y}=c}
$$

They are in fact the effective chemical potentials at $0 \mathrm{~K}$, and determine the preference of the alloy components to occupy site $\alpha$ relative to that of sublattice E. In general, the pair and higher order interaction parameters could also be taken into account. However, as we have shown in [16], the on-site interaction parameters are dominant in the total energy expansion. The summation in Equation (3) runs only over four sublattices, since the occupation at the fifth sublattice is determined by the alloy composition $c$ and the concentrations at the other four sublattices $c_{\alpha} . E_{0}(c)$ is the total energy of a homogeneous random alloy $\left(c_{\alpha}=c\right)$ and $J_{\alpha}^{(1)}$ are the on-site interaction parameters.

The second term in Equation (1) contains the configurational and magnetic entropy correspondingly given by:

$$
S_{\text {conf }}=-k_{B} \sum_{\alpha=1,5} n_{\alpha}\left[c_{\alpha} \ln c_{\alpha}+\left(1-c_{\alpha}\right) \ln \left(1-c_{\alpha}\right)\right]
$$


and

$$
S_{\text {magn }}=k_{B} \sum_{\alpha=1,5} n_{\alpha}\left[c_{\alpha} \ln \left(1+\mu_{\alpha}^{A}\right)+\left(1-c_{\alpha}\right) \ln \left(1+\mu_{\alpha}^{B}\right)\right]
$$

Here $\mu_{\alpha}$ are the local magnetic moments of the alloy components $(A, B)$ in the $A-B \sigma$-phase, which are found by minimizing the corresponding magnetic free energy in the first-principles calculations at a given temperature.

Although the $\sigma$-phase forms at high temperatures in the paramagnetic state, the local magnetic moments do not disappear on the Fe atoms, as has been shown by Korzhavyi et al. [7] for the $\mathrm{FeCr} \sigma$-phase, and they significantly contribute to the energetics of the $\sigma$-phase. Furthermore, as has already been discussed in our previous works $[14,16]$, longitudinal spin fluctuations should exist at high temperature. These are thermal Stoner-like many-body excitations which lead to fluctuations of the magnitude of the local magnetic moments. In order to calculate these high temperature magnetic excitations we adopt a simple model [17], which implies that longitudinal spin fluctuations (LSF) contribute to the magnetic entropy of the paramagnetic state. The LSFs induce modified spin magnetic moments on the alloy components, while for instance the $\mathrm{Cr}$ atoms would have zero magnetic moments in the disordered local moment (DLM) state.

In present work we assume that the lattice vibrations do not play a decisive role in the distribution of the alloy components between the sublattices and therefore neglect their contribution in Equation (1). Furthermore, since the $\sigma$-phase forms at relatively high temperatures and there is no experimental evidence of being close to any kind of phase transition (ordering or magnetic), we also neglect contributions from short-range order effects in the configurational part.

\section{First-Principles Calculations}

The total energies of random alloys and the on-site interaction parameters in Equation (3) have been obtained by the exact muffin-tin orbital (EMTO) method using the full charge density formalism (FCD) $[18,19]$. The EMTO method is considered as an improved screened Korringa-Kohn-Rostoker method, which implies large overlapping potential spheres for a sufficiently accurate description of the exact single-electron potential, while the FCD method was devised to perform with high efficiency as well as with an accuracy close to full potential total energy methods [20]. In the present work the exchange-correlation functional was evaluated within the generalized gradient approximation [21].

The electronic structure of the random alloy in the $\sigma$-phase has been calculated using the coherent potential approximation (CPA) [22]. We have demonstrated in [16] that the density of states and the average magnetic moments are well reproduced by the CPA. The screened Coulomb interactions have been chosen to be $\alpha_{s c r}=0.67$ and $\beta_{s c r}=1.05$ for all the $\sigma$-phase sublattices. These values are approximate, but the corresponding supercell calculations, which would be needed to evaluate all screening constants, are impractical.

Local Lattice Relaxations

In our previous calculations for the $\mathrm{FeCr} \sigma$-phase, we have neglected the effect of local atomic relaxations. As has been shown in [14], the contribution from local lattice relaxations to the formation 
enthalpy is about $2.5 \mathrm{mRy} / 30$ at. cell in the $\mathrm{FeCr}$ case, which was expected due to small size mismatch of $\mathrm{Fe}$ and $\mathrm{Cr}$. In case of the $\mathrm{CoCr}, \mathrm{ReW}$ and $\mathrm{FeV} \sigma$-phases we show in Table 1 the results for the energy of local lattice relaxations $E_{\text {relax }}$, which have been obtained for the room temperature values of an unit cell volume $V$ and a ratio of the lattice cell constants $c / a$ by the full-potential linearized augmented plane wave method [23], implemented in the WIEN2k code [24]. Obviously, $E_{\text {relax }}$ is small in the case of the $\mathrm{CoCr}$ and $\operatorname{ReW} \sigma$-phases, but not negligible in the case of the $\mathrm{FeV} \sigma$-phase.

Table 1. The room temperature lattice parameters $(V, c / a)$, the corresponding size (atomic radii) mismatch of the alloy components $\Delta_{A R}$ and the energy of the local atomic relaxations $E_{\text {relax }}$ in the $\mathrm{Re}_{0.5} \mathrm{~W}_{0.5}, \mathrm{Co}_{0.5} \mathrm{Cr}_{0.5}$ and $\mathrm{Fe}_{0.5} \mathrm{~V}_{0.5} \sigma$-phases.

\begin{tabular}{lcccc}
\hline System & Volume & $c / a$ & $\boldsymbol{\Delta}_{\boldsymbol{A R}}$ & $\boldsymbol{E}_{\text {relax }}$ \\
\hline $\mathrm{Re}_{0.5} \mathrm{~W}_{0.5}$ & $0.46482 \mathrm{~nm}^{3} a$ & $0.521^{a}$ & $0.002 \mathrm{~nm}$ & $5 \mathrm{mRy} / 30$ at. cell \\
$\mathrm{Co}_{0.5} \mathrm{Cr}_{0.5}$ & $0.35235 \mathrm{~nm}^{3}{ }^{b}$ & $0.517^{b}$ & $0.003 \mathrm{~nm}$ & $6 \mathrm{mRy} / 30$ at. cell \\
$\mathrm{Fe}_{0.5} \mathrm{~V}_{0.5}$ & $0.37074 \mathrm{~nm}^{3} c$ & $0.517^{c}$ & $0.008 \mathrm{~nm}$ & $30 \mathrm{mRy} / 30$ at. cell \\
\hline \multicolumn{5}{c}{ Ref. [25]; ${ }^{b}$ Ref. [26]; ${ }^{c}$ Ref. [27]. }
\end{tabular}

In order to account for the effects of the lattice relaxations in the Fe- $\mathrm{V}$ system, we introduce in our model an additional term, called $J_{\alpha}^{(\text {relax })}$, such that the total energy has the following form:

$$
E_{\text {tot }}\left(\left\{c_{\alpha}\right\}\right)=E_{0}(c)+\sum_{\alpha=1,4(A, D)} n_{\alpha}\left(J_{\alpha}^{(1)}+J_{\alpha}^{(\text {relax })}\right) c_{\alpha}
$$

These $J_{\alpha}^{(\text {relax })}$ parameters can be interpreted as the energy gain due to the atomic relaxations with respect to substitution of one Fe atom by one $\mathrm{V}$ atom in each sublattice $\alpha$. To calculate these energies we use the WIEN2k code and simulate a cell of 30 atoms, in which we exchange one Fe atom from each of the A, B, C and D sublattices with one $\mathrm{V}$ atom from the $\mathrm{E}$ sublattice. Thus the relaxation parameter is defined as $J_{\alpha}^{(\text {relax })}=E_{\alpha}^{(\text {relax })}-E_{E}^{(\text {relax })}$, where $E_{\alpha}^{(\text {relax })}$ is the energy of relaxation of $\alpha=\mathrm{A}, \mathrm{B}, \mathrm{C}$ and D site with respect to substitution of one Fe atom and $E_{E}^{(\text {relax })}$ is the energy of relaxation of the E site with respect to substitution of one $\mathrm{V}$ atom.

\section{Results and Discussion}

\subsection{The Fe-Cr $\sigma$-Phase}

In $[14,16]$ we analyzed the possible effects, which may play a role in the behaviour of the atomic site distribution in the $\sigma$-phase. These are the magnetic state, structural variations (volume and $c / a$ ), which might be present in the system due to effects of the neutron irradiation or thermal expansion, and temperature effects. It was found that the magnetic entropy contributes mainly to the icosahedral coordinated (A, D) and highly coordinated B sites. Usually these sites are predominantly occupied by only one sort of the alloy components. At the same time the structural variations lead to an additional atomic redistribution. In particular, a volume expansion promotes an accumulation of more $\mathrm{Cr}$ in the $\mathrm{A}$ and $\mathrm{D}$ sublattices, while the $c / a$ ratio affects the occupation of the $\mathrm{C}$ sites. 
Taking these effects into account, we have calculated the $\mathrm{Cr}$ site occupancies in the $\mathrm{Fe}_{0.5} \mathrm{Cr}_{0.5} \sigma$-phase for the low and high temperature values that were obtained for the experimental room temperature lattice parameters and temperature expanded ones. The results are shown in Figure 2 and agree quite well with the available experimental data $[2,28]$. At very high temperatures the configurational entropy drives the system to a highly disordered distribution of the alloy components on the five $\sigma$-phase sublattices. By decreasing the temperature, $\mathrm{Fe}$ and $\mathrm{Cr}$ atoms exhibit a clear preference for different inequivalent sites. At temperatures of about $700 \mathrm{~K}$, which is the lower border of the $\sigma$-phase stability range, the (A, D) sites become occupied only by Fe atoms (i.e., nearly no $\mathrm{Cr}$ atoms), the (B, E) sites mostly by $\mathrm{Cr}$ atoms, while the occupation of the $\mathrm{C}$ site remains almost unchanged and mixed. It is interesting to note that the preference of the $\mathrm{Cr}$ atoms between the $\mathrm{B}$ and $\mathrm{E}$ sites crosses around 650-700 K, at a temperature where the $\sigma$-phase formation starts. The occupation behavior for other sites also changes at this temperature as discussed above. This effect might be due to structural variations with temperature in our model, whereas no experimental data on thermal expansion of the $\sigma$-phase in any system is found in literature.

Figure 2. The $\mathrm{Cr}$ site occupancies of the (A, B, C, D, E) sublattices versus temperature including the magnetic and temperature effects in the $\mathrm{Fe}_{0.5} \mathrm{Cr}_{0.5} \sigma$-phase. The empty symbols denote the available experimental data (error bars within the symbol sizes) [2,28]. The vertical lines indicate the temperature range of the $\sigma$-phase formation in the $\mathrm{Fe}-\mathrm{Cr}$ alloy. (Reprinted figure with permission from [16] as follows: Kabliman, E.; Blaha, P.; Schwarz, K.; Peil, O.E.; Ruban, A.V.; Johansson, B. Phys. Rev. B 2011, 84, 184206. Copyright (2011) by the American Physical Society. http://link.aps.org/abstract/PRB/v84/p184206).

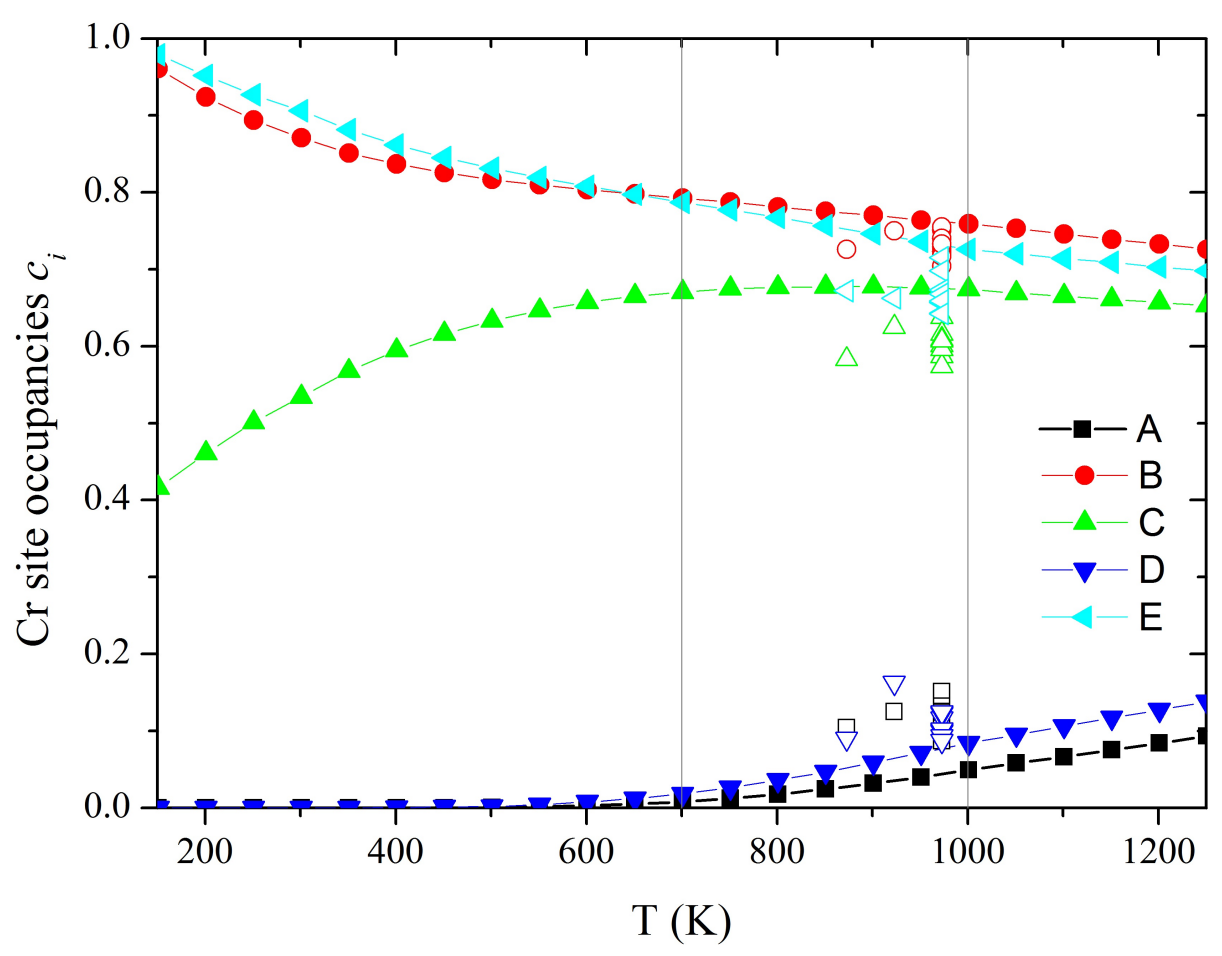




\subsection{The Re-W and $\mathrm{Co}-\mathrm{Cr} \sigma$-Phase}

In order to test the applicability of our model to other binary $\sigma$-phases we first chose the Re-W and $\mathrm{Co}-\mathrm{Cr}$ systems, which have a small mismatch between the atomic radii similar to the $\mathrm{Fe}-\mathrm{Cr}$ system. The calculated effective on-site interactions and magnetic moments of these two systems are given in Table 2. According to [29] the Co-Cr system is a possible third candidate for a magnetic $\sigma$-phase in addition to the $\mathrm{Fe}-\mathrm{Cr}$ and $\mathrm{Fe}-\mathrm{V} \sigma$-phases. The magnetism of the $\mathrm{Co}-\mathrm{Cr} \sigma$-phase at $0 \mathrm{~K}$ was theoretically studied in [10]. In present work it was found that both the $\mathrm{Co}$ and $\mathrm{Cr}$ atoms in the $\mathrm{Co}-\mathrm{Cr} \sigma$-phase have finite magnetic moments, even at the relevant high temperatures. In contrary the Re-W system is non-magnetic. Using these parameters the distributions of the alloy components in the $\mathrm{Re}_{0.5} \mathrm{~W}_{0.5}$ and $\mathrm{Co}_{0.5} \mathrm{Cr}_{0.5}$ alloys were calculated and the corresponding results are shown in Figure 3 in comparison with the $\mathrm{Fe}_{0.5} \mathrm{Cr}_{0.5} \sigma$-phase. Taking into account the small mismatch between the atomic radii of the alloy components in the Re-W and Co-Cr systems and the absence of corresponding experimental data, in the present work we neglect structural changes with respect to the alloy composition and temperature within the range of the $\sigma$-phase formation. Nevertheless these effects can be approximately estimated based on the corresponding study of the $\mathrm{Fe}-\mathrm{Cr} \sigma$-phase.

Table 2. On-site parameters $J_{\alpha}^{(1)}$ (mRy/cell) calculated for the $\mathrm{Re}_{0.5} \mathrm{~W}_{0.5}$ and $\mathrm{Co}_{0.5} \mathrm{Cr}_{0.5}$ systems within the DLM-LSF model at $T=1,400 \mathrm{~K}$ and $T=1,500 \mathrm{~K}$ respectively. $\mu_{\alpha}^{C o}$ and $\mu_{\alpha}^{C r}$ are the local magnetic moments of the $\mathrm{Co}$ and $\mathrm{Cr}$ atoms in the $\mathrm{Co}_{0.5} \mathrm{Cr}_{0.5} \sigma$-phase, while the Re-W $\sigma$-phase is nonmagnetic.

\begin{tabular}{lcccccc}
\hline & & $\mathbf{A}$ & $\mathbf{B}$ & $\mathbf{C}$ & $\mathbf{D}$ & $\mathbf{E}$ \\
\hline$J_{\alpha}^{(1)}(\mathrm{mRy} / \mathrm{cell})$ & $\mathrm{ReW}$ & 15.188 & -8.156 & -1.594 & 12.656 & 0.0 \\
& $\mathrm{CoCr}$ & 15.656 & -1.500 & 0.563 & 12.656 & 0.0 \\
$\mu_{\alpha}^{C o}\left(\mu_{B}\right)$ & $\mathrm{Co}$ & 0.802 & 1.167 & 1.064 & 0.854 & 0.875 \\
$\mu_{\alpha}^{C r}\left(\mu_{B}\right)$ & $\mathrm{Cr}$ & 0.491 & 0.903 & 0.673 & 0.570 & 0.564 \\
\hline
\end{tabular}

As can be seen from Figure 3 the site occupancies in the $\mathrm{Re}_{0.5} \mathrm{~W}_{0.5}$ system behave similarly to the $\mathrm{Fe}_{0.5} \mathrm{Cr}_{0.5}$ alloy. However, one can notice that at low temperatures the $\mathrm{C}$ and $\mathrm{E}$ sites in the $\mathrm{Re}_{0.5} \mathrm{~W}_{0.5}$ $\sigma$-phase are occupied by the $\mathrm{W}$ atoms in reverse order in comparison to the $\mathrm{Cr}$ atoms in the $\mathrm{Fe}_{0.5} \mathrm{Cr}_{0.5}$ $\sigma$-phase. This is in agreement with earlier published results [30,31]. Both theoretical results agree well with the experimental data from [30]. The reversed occupation of the $\mathrm{C}$ and $\mathrm{E}$ sites seems to be caused by the big difference between the $\mathrm{Cr}$ and $\mathrm{W}$ atomic radii. Thus the occupation of these sites ( $\mathrm{C}$ and $\mathrm{E})$ can change with respect to each other depending on the alloy component.

For the $\mathrm{Co}_{0.5} \mathrm{Cr}_{0.5} \sigma$-phase the values of the $\mathrm{Cr}$ site occupancies are almost the same as in the $\mathrm{Fe}_{0.5} \mathrm{Cr}_{0.5}$ system, but the slightly pronounced dominance of a particular alloy component in the occupation of a particular site occurs and can be explained by the slightly larger size mismatch between the alloy components in the $\mathrm{Co}-\mathrm{Cr}$ than in $\mathrm{Fe}-\mathrm{Cr}$ system. The $\mathrm{Co}-\mathrm{Cr} \sigma$-phase forms more on the right hand side of the phase diagram (larger $\mathrm{Cr}$ content) and is usually observed for a concentration range $54 \%-67 \%$ of $\mathrm{Cr}$ in a temperature interval $600-1,280{ }^{\circ} \mathrm{C}$ [29]. As in the Fe-Cr system (see $\left.[14,16]\right)$, one can use the parameters obtained for the $\mathrm{Co}_{0.5} \mathrm{Cr}_{0.5}$ in order to predict the $\mathrm{Cr}$ site occupancies in the 
full composition range of the $\sigma$-phase formation. In Figure 4 the $\mathrm{Cr}$ site distribution calculated versus the alloy composition is displayed together with the experimental data from [29] measured at various temperatures. Taking into account the scattering of the experimental data, good agreement is found between the present calculated results and the experimental measurements (probabilities), justifying our theoretical model also for this case.

Figure 3. The site occupancies of the (A, B, C, D, E) sublattices for the reference components in the $\mathrm{Re}_{0.5} \mathrm{~W}_{0.5}, \mathrm{Co}_{0.5} \mathrm{Cr}_{0.5}$ and $\mathrm{Fe}_{0.5} \mathrm{~V}_{0.5}$ alloys in comparison with the results for the $\mathrm{Fe}_{0.5} \mathrm{Cr}_{0.5} \sigma$-phase from [14] (without temperature effects).

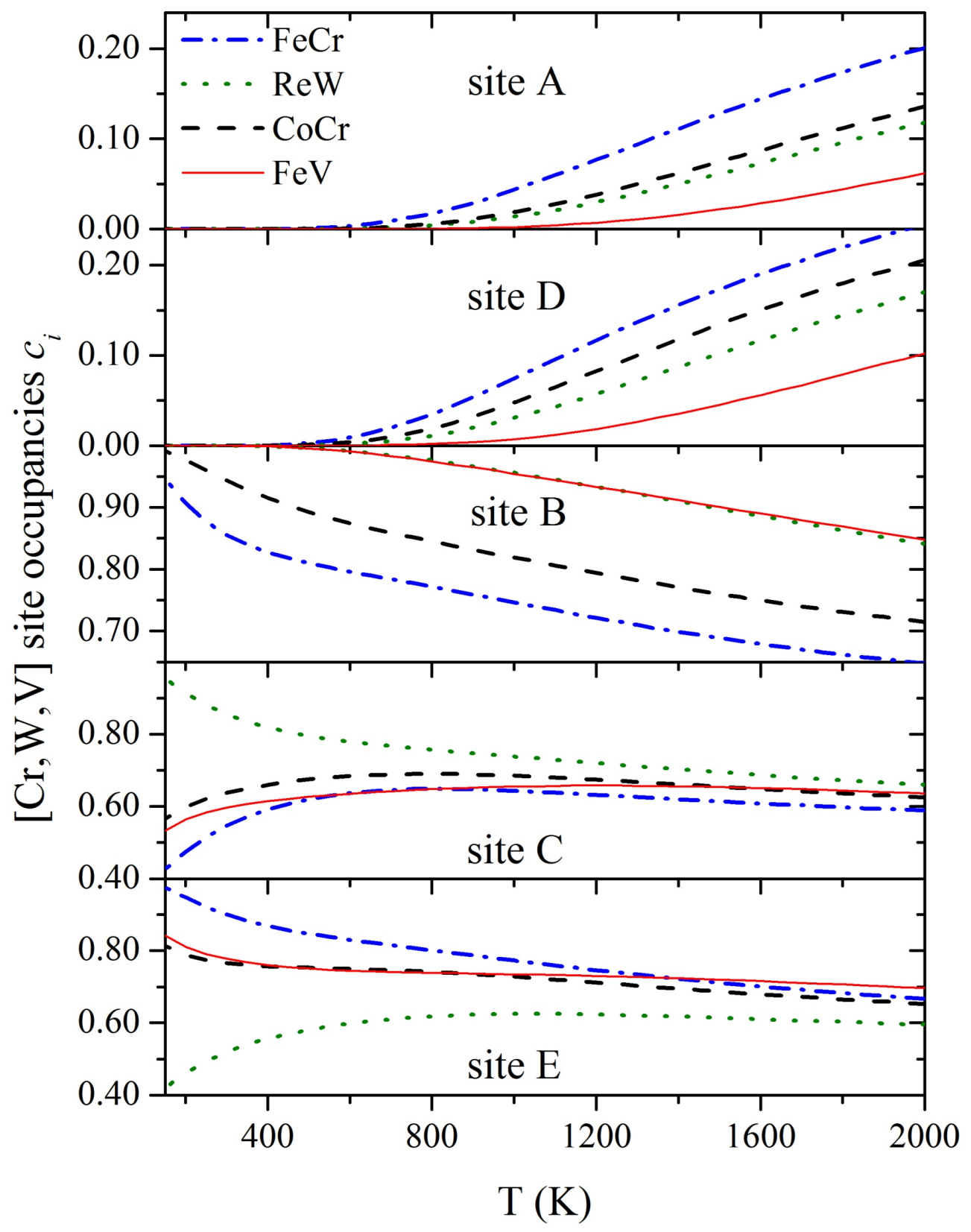


Figure 4. The $\mathrm{Cr}$ site occupancies of the (A, B, C, D, E) sublattices in the Co-Cr system versus the $\mathrm{Cr}$ composition, $x_{C r}$, at fixed $T=1,500 \mathrm{~K}$. The full symbols denote the calculated results, the empty symbols represent the experimental site occupancies (error bars are less than $0.5 \%$ ) [29].

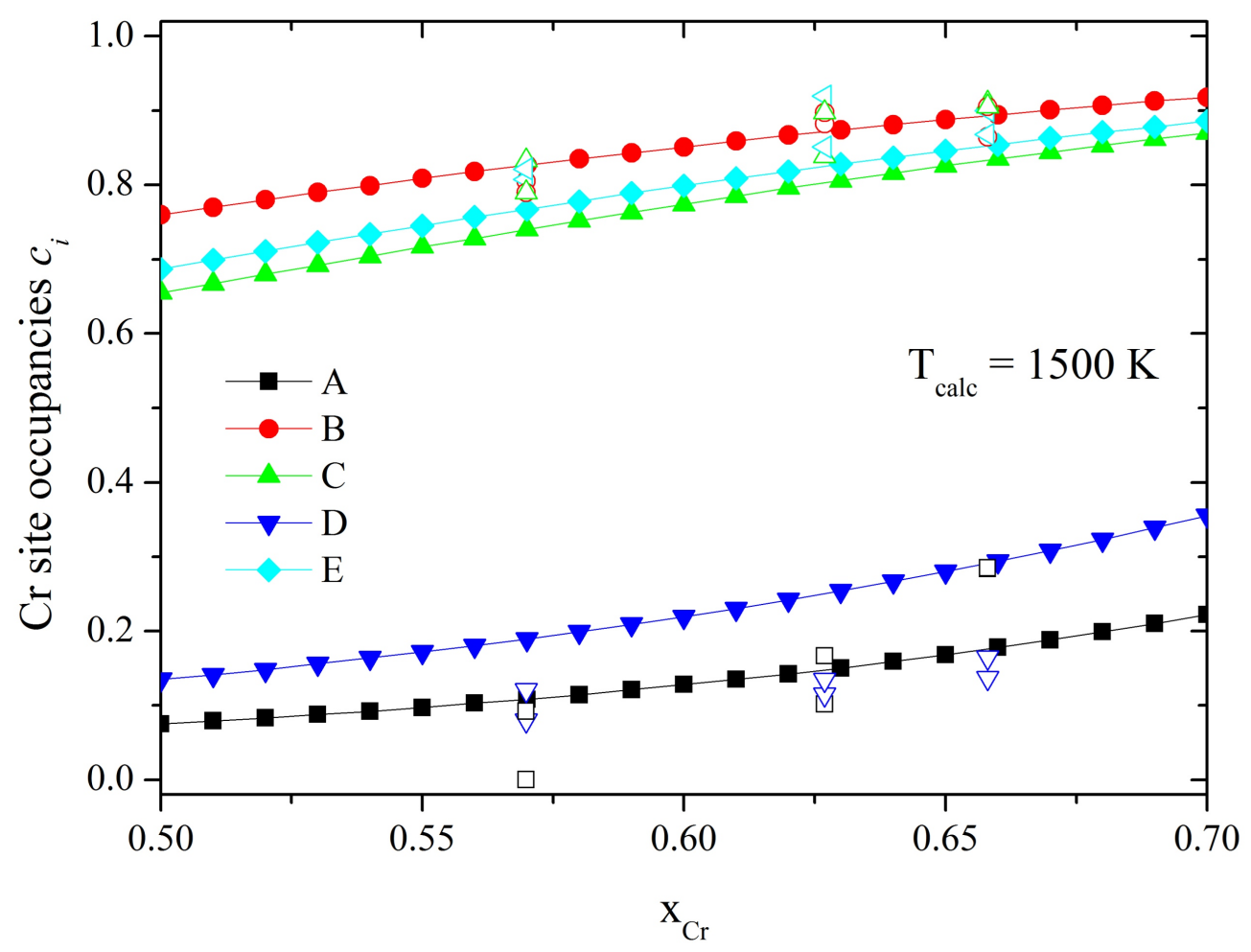

\subsection{The Fe-V Sigma-Phase}

The formation probability of the $\sigma$-phase in the $\mathrm{Fe}-\mathrm{V}$ alloy is many times larger than in the $\mathrm{Fe}-\mathrm{Cr}$ system due to a much wider temperature and compositional ranges, where this phase is stable [32]. Despite the fact that the $\sigma$-phase in the Fe-V system was found many years ago, the concentration and temperature ranges of its existence are still not precisely known. In general it is difficult to accurately determine the phase boundaries due to several factors such as: the slow kinetics of the transformation, the dependence on the purity of the alloys, the mechanical state (strained or strain-free state) and the annealing temperature. According to the phase diagrams analyzed in [32] the upper temperature limit for the $\sigma$-phase stability equals to $1,321^{\circ} \mathrm{C}$ for an alloy composition $x_{V}=46.5 \%$, and it depends rather strongly on composition: $\sim 1,000^{\circ} \mathrm{C}$ for $x_{V}=34 \%$ and $\sim 1,050{ }^{\circ} \mathrm{C}$ for $x_{V}=63 \%$. On the other hand, the lower temperature limit of formation does not depend too much on the concentration and is around $647-650{ }^{\circ} \mathrm{C}$.

\section{Local Atomic Relaxations}

As mentioned above, the quite large size mismatch of the alloy components in the Fe- $\mathrm{V}$ system causes that the effect of local atomic relaxations might not be negligible in contrast to the other studied systems (Fe-Cr, Re-W and Co-Cr) (see Table 1). In order to account for these effects we introduced a new parameter in our model, namely $J_{\alpha}^{(\text {relax })}$, which we calculated for each sublattice in the $\mathrm{Fe}_{0.5} \mathrm{~V}_{0.5}$ 
$\sigma$-phase (assuming the experimental lattice parameters $V=0.37074 \mathrm{~nm}^{3}$ and $c / a=0.517$ ) and list them in Table 3. As can be seen, the local lattice relaxations change the effective on-site interactions only moderately and consequently the corresponding site occupancies will be hardly affected. Therefore one might expect that the effect of local atomic relaxations in binary $\sigma$-phases near the equiatomic composition is negligible for all binary systems, for which the component size mismatch is less than that in the Fe-V system.

Table 3. On-site interaction parameters $J_{\alpha}^{(1)}$ (mRy/cell) calculated for the $\mathrm{Fe}_{0.5} \mathrm{~V}_{0.5}$ alloy with/without taking into account local lattice relaxations $J_{\alpha}^{(\text {relax })}$ for the experimental lattice parameters $V=0.37074 \mathrm{~nm}^{3}$ and $c / a=0.517$ within the DLM-LSF model at $T=1,000 \mathrm{~K}$.

\begin{tabular}{lccccc}
\hline & A & B & C & D & E \\
\hline$J_{\alpha}^{(1)}($ mRy/cell $)$ & 24.750 & -7.594 & 0.656 & 20.344 & 0.0 \\
$J_{\alpha}^{(1)}+J_{\alpha}^{(\text {relax })}($ mRy/cell $)$ & 22.594 & -7.219 & 0.656 & 18.656 & 0.0 \\
\hline
\end{tabular}

Site Occupancies $\left(x_{V}=0.50\right)$

Let us start from the equiatomic composition $x_{V}=0.50$ and consider a possible increase in volume from $V_{\text {exp }}=0.37074 \mathrm{~nm}^{3}$ to the high temperature value $V=0.38319 \mathrm{~nm}^{3}$ due to thermal expansion. The calculated effective on-site interactions and corresponding local magnetic moments of Fe and $\mathrm{V}$ atoms are given in Table 4 at three different temperatures.

Table 4. On-site parameters $J_{\alpha}^{(1)}$ (mRy/cell) and local magnetic moments of Fe and V atom $\mu_{\alpha}^{F e}$ and $\mu_{\alpha}^{V}$ calculated for the lattice parameters $V=0.38319 \mathrm{~nm}^{3}$ and $c / a=0.517$ within the DLM-LSF model at three different temperatures $T=500,1,000$ and 1,500 K.

\begin{tabular}{lcccccc}
\hline & $\mathbf{T}(\mathbf{K})$ & $\mathbf{A}$ & $\mathbf{B}$ & $\mathbf{C}$ & $\mathbf{D}$ & $\mathbf{E}$ \\
\hline$J_{\alpha}^{(1)}(\mathrm{mRy} / \mathrm{cell})$ & 500 & 25.406 & -7.125 & 1.031 & 21.000 & 0.0 \\
& 1,000 & 24.750 & -7.594 & 0.656 & 20.344 & 0.0 \\
& 1,500 & 24.094 & -7.781 & 0.375 & 19.875 & 0.0 \\
& & & & & & \\
$\mu_{\alpha}^{F e}\left(\mu_{B}\right)$ & 500 & 0.841 & 1.768 & 1.445 & 1.089 & 0.895 \\
& 1,000 & 1.047 & 1.790 & 1.518 & 1.240 & 1.079 \\
& 1,500 & 1.169 & 1.804 & 1.571 & 1.334 & 1.187 \\
$\mu_{\alpha}^{V}\left(\mu_{B}\right)$ & & & & & & \\
& 500 & 0.091 & 0.119 & 0.108 & 0.098 & 0.091 \\
& 1,000 & 0.170 & 0.217 & 0.200 & 0.183 & 0.171 \\
& 1,500 & 0.240 & 0.303 & 0.280 & 0.257 & 0.242 \\
\hline
\end{tabular}

Using the $J_{\alpha}^{(1)}$ parameters and the corresponding $\mathrm{Fe}$ and $\mathrm{V}$ magnetic moments (obtained at $T=1,000 \mathrm{~K}$ ), we calculated the distribution of the $\mathrm{V}$ atoms among the five $\sigma$-phase sublattices in the 
$\mathrm{Fe}_{0.5} \mathrm{~V}_{0.5}$ alloy with respect to temperature and alloy composition (see Figure 5). The results obtained at high temperatures agree reasonably well with the experimental values measured for an alloy composition $x_{V}=0.48$. At the low temperature we find that for example the $\mathrm{C}$ sublattice is equally occupied by both alloy components. Using the WIEN2k code we also calculated the equilibrium atomic distribution at $T=0 \mathrm{~K}$. In contrast to the $\mathrm{Fe}_{0.5} \mathrm{Cr}_{0.5}$ alloy (see Figure 3), the E sublattice of the $\mathrm{Fe}_{0.5} \mathrm{~V}_{0.5} \sigma$-phase has a partial site occupancy by $\mathrm{V}$ atoms. There are $7 \mathrm{~V}+1 \mathrm{Fe}$ atoms in the $\mathrm{E}$ sublattice, while the $\mathrm{C}$ sublattice has an equal occupancy by both alloy components, i.e., $4 \mathrm{~V}+4 \mathrm{Fe}$ atoms. Thus the atomic site occupancies estimated within our model at low temperatures are found in good agreement with the ground state equilibrium distribution obtained at $T=0 \mathrm{~K}$ by the WIEN2k code.

Figure 5. The V site occupancies of the (A, B, C, D, E) sublattices versus the V composition, $x_{V}$, at fixed $T=1,000 \mathrm{~K}$. The full symbols denote the calculated results, the empty symbols represent the available experimental data (error bars within the symbol sizes) [28,32]. $J_{\alpha}^{(1)}$ and $\mu_{\alpha}^{(F e, V)}$ are obtained for $\mathrm{Fe}_{0.5} \mathrm{~V}_{0.5}$ at $T=1,000 \mathrm{~K}$.

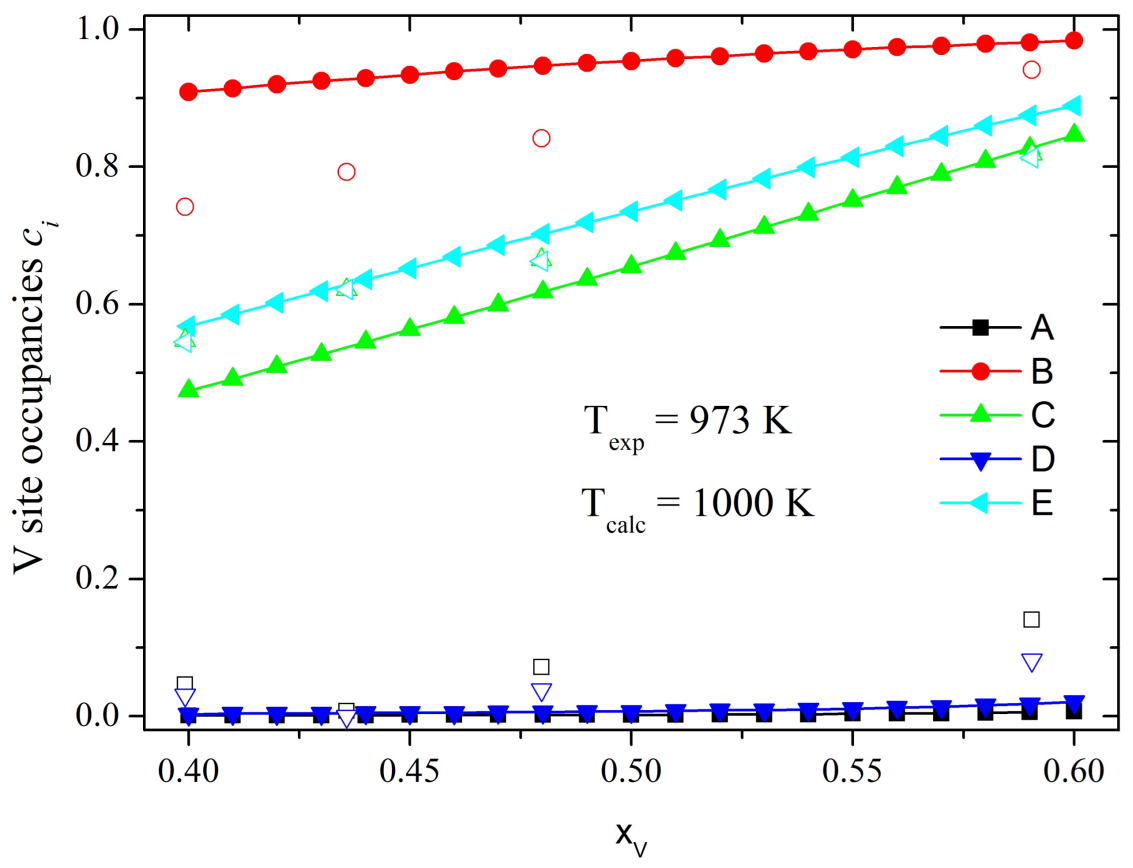

Site Occupancies $\left(x_{V}=0.40,0.60\right)$

Let us now consider the atomic site distribution in the Fe-V $\sigma$-phase with respect to the alloy composition, $x_{V}$ (see Figure 5). The predicted site occupancies as function of the alloy composition reproduce well the experimental values for the $\mathrm{C}$ and $\mathrm{E}$ sublattices, while they differ in slope for the $\mathrm{B}$ and (A, D) sublattices. Due to the large size mismatch between the Fe and V atoms, the lattice parameters of the $\mathrm{Fe}-\mathrm{V} \sigma$-phase should depend significantly on the alloy composition. According to experimental measurements [27] an increase of the vanadium content by $10 \%$ from the equiatomic composition, $\mathrm{Fe}_{0.5} \mathrm{~V}_{0.5}$, linearly increases the volume of the unit cell from $0.37074 \mathrm{~nm}^{3}$ (for $x_{V}=0.5$ ) to $0.37634 \mathrm{~nm}^{3}$ (for $x_{V}=0.6$ ), while the $c / a$ values decrease from 0.517 to 0.516 , respectively. On the other hand, with a decrease of the vanadium content by $10 \%$ to $x_{V}=0.4$, the volume and $c / a$ ratio 
change to $0.36514 \mathrm{~nm}^{3}$ and 0.518 , correspondingly. Assuming a linear dependence, one can extrapolate the expanded volumes as $V=0.37747 \mathrm{~nm}^{3}$ for $x_{V}=0.4$ and $V=0.38892 \mathrm{~nm}^{3}$ for $x_{V}=0.6$. The optimization of the $c / a$ ratio in the Fe- $\mathrm{V} \sigma$-phase within the EMTO-CPA method is not really reliable due to the large size mismatch of the Fe and $\mathrm{V}$ atoms. Therefore, we stay at a constant $c / a$ ratio, but keep in mind that the site occupancies might vary due to a change of $c / a$, as has been discussed for the $\mathrm{Fe}-\mathrm{Cr} \sigma$-phase.

The corresponding effective interactions $J_{\alpha}^{(1)}$ and magnetic moments $\mu_{\alpha}^{(F e, V)}$ obtained for $x_{V}=0.40$ and $x_{V}=0.60$ are given in Table 5. One can see that for $x_{V}=0.40$ all four effective interactions are different (smaller in absolute values) from those obtained for $x_{V}=0.50$, while for $x_{V}=0.60$ only the changes for the $\mathrm{B}$ and $\mathrm{C}$ sublattices are remarkable. Nevertheless, when we compare the site occupancies for the parameters obtained for $x_{V}=0.40$ and $x_{V}=0.60$ with those predicted from the parameters obtained for $x_{V}=0.50$, only small differences can be observed. The corresponding values of the site occupancies calculated at $T=1,000 \mathrm{~K}$ are given in Table 6 . As can be seen only the $\mathrm{B}, \mathrm{C}$ and $\mathrm{E}$ site occupancies change noticeably (those, which have predominant occupancy by $\mathrm{V}$ atoms) for $x_{V}=0.40$, while for $x_{V}=0.60$ all values are nearly the same. For $x_{V}=0.40$ the $\mathrm{C}$ and $\mathrm{E}$ sublattices now have almost an equal occupation in accord with the experimental data (see Figure 5). The occupancy of the B site is a bit smaller, but still higher than the corresponding experimental value. This discrepancy could be due to several reasons: additional structural variations with respect to the alloy composition, temperature expansion, or local atomic relaxations. Particularly the latter might not be negligible for alloy compositions different from the equiatomic composition, especially for those sublattices with small deviations from integer occupation, e.g., the A and D sublattices for $x_{V}=0.60$ or the B sublattice for $x_{V}=0.40$. These effects could be taken into account similiar as for the equiatomic composition (see Equation (6)), but such calculations are not presented in this work.

Table 5. On-site parameters $J_{\alpha}^{(1)}$ (mRy/cell) and local magnetic moments of Fe and V, $\mu_{\alpha}^{F e}$ and $\mu_{\alpha}^{V}$, calculated for different alloy compositions $x_{V}=\{0.40 ; 0.50 ; 0.60\}$ in the Fe-V system with the corresponding lattice parameters $V=\{0.37747 ; 0.38319 ; 0.38892\} \mathrm{nm}^{3}$ and $c / a=\{0.518 ; 0.517 ; 0.516\}$ within the DLM-LSF model at $T=1,000 \mathrm{~K}$.

\begin{tabular}{lcccccc}
\hline & $\boldsymbol{x}_{\boldsymbol{V}}$ & $\mathbf{A}$ & $\mathbf{B}$ & $\mathbf{C}$ & $\mathbf{D}$ & $\mathbf{E}$ \\
\hline$J_{\alpha}^{(1)}(\mathrm{mRy} / \mathrm{cell})$ & 0.40 & 23.625 & -7.031 & -0.094 & 19.406 & 0.0 \\
& 0.50 & 24.750 & -7.594 & 0.656 & 20.344 & 0.0 \\
& 0.60 & 24.938 & -8.625 & 0.844 & 20.531 & 0.0 \\
& & & & & & \\
$\mu_{\alpha}^{F e}\left(\mu_{B}\right)$ & 0.40 & 1.158 & 1.908 & 1.659 & 1.337 & 1.265 \\
& 0.50 & 1.047 & 1.790 & 1.518 & 1.240 & 1.079 \\
& 0.60 & 0.947 & 1.644 & 1.355 & 1.148 & 0.908 \\
$\mu_{\alpha}^{V}\left(\mu_{B}\right)$ & & & & & & \\
& 0.40 & 0.168 & 0.221 & 0.199 & 0.181 & 0.172 \\
& 0.50 & 0.170 & 0.217 & 0.200 & 0.183 & 0.171 \\
& 0.60 & 0.171 & 0.213 & 0.210 & 0.184 & 0.169 \\
\hline
\end{tabular}


Table 6. The $\mathrm{V}$ site occupancies in the Fe-V system calculated for different alloy compositions $x_{V}=\{0.40 ; 0.60\}$ with parameters obtained for the fixed $\mathrm{Fe}_{0.5} \mathrm{~V}_{0.5}$ composition or taking into account concentration and structural effects as described in the text within the DLM-LSF model at $T=1,000 \mathrm{~K}$.

\begin{tabular}{ccccccc}
\hline $\boldsymbol{x}_{\boldsymbol{V}}$ & $(\boldsymbol{V} ; \boldsymbol{c} / \boldsymbol{a})$ & $\mathbf{A}$ & $\mathbf{B}$ & $\mathbf{C}$ & $\mathbf{D}$ & $\mathbf{E}$ \\
\hline 0.40 & $\left(0.38319 \mathrm{~nm}^{3} ; 0.517\right)$ & 0.001 & 0.917 & 0.473 & 0.002 & 0.567 \\
0.40 & $\left(0.37747 \mathrm{~nm}^{3} ; 0.518\right)$ & 0.001 & 0.897 & 0.511 & 0.002 & 0.538 \\
& & & & & & \\
0.50 & $\left(0.38319 \mathrm{~nm}^{3} ; 0.517\right)$ & 0.001 & 0.959 & 0.656 & 0.004 & 0.735 \\
& & & & & & \\
0.60 & $\left(0.38319 \mathrm{~nm}^{3} ; 0.517\right)$ & 0.003 & 0.986 & 0.851 & 0.013 & 0.893 \\
0.60 & $\left(0.38892 \mathrm{~nm}^{3} ; 0.516\right)$ & 0.003 & 0.990 & 0.846 & 0.012 & 0.896 \\
\hline
\end{tabular}

\section{Summary}

In present work we gave a short overview of a single-site mean field theory for calculating of the atomic site occupancies in the $\sigma$-phase. This method was applied to the $\mathrm{Fe}-\mathrm{Cr}, \mathrm{Re}-\mathrm{W}, \mathrm{Co}-\mathrm{Cr}$ and $\mathrm{Fe}-\mathrm{V}$ systems. It was shown that the chosen binary $\sigma$-phases have similar preferences in occupation of the particular lattice sites except for the Re-W system, where a reversal occupation of two sites was observed in agreement with previously published works. For one system, namely Fe-V, which has a large atomic mismatch between the alloy components, we included into our model an additional parameter, which describes the effects of local atomic relaxations. The results obtained in the present work were found in a good agreement with the experimental data and previously published theoretical works. The suggested model can in principle be generalized for multicomponent systems.

\section{Acknowledgments}

This work was partly supported by the Austrian Science Fund (FWF) within the Science College CMS (Grant W004) and the special research program ViCoM (Grant F41). A.V.R. is grateful to the Swedish Research Council (VR) and Hero-m centrum, Jernkontoret, and ERC grant for financial support.

\section{References}

1. Garin, J.L.; Mannheim, R.L. Sigma-phase precipitation upon industrial-like heating of cast heat-resistant steels. J. Mater. Process. Technol. 2009, 209, 3143-3148.

2. Yakel, H.L. Atom distributions in sigma phases. I. Fe and $\mathrm{Cr}$ atom distributions in a binary sigma phase equilibrated at 1063, 1013 and 923 K. Acta Cryst. B 1983, 39, 20-28.

3. Frank, F.C.; Kasper, J.S. Complex alloy structures regarded as sphere packings. I. Definitions and basic principles. Acta Cryst. 1958, 11, 184-190.

4. Kasper, J.S.; Waterstrat, R. Ordering of atoms in the $\sigma$-phase. Acta Cryst. 1956, 9, 289-295. 
5. Sluiter, M.H.F.; Esfarjani, K.; Kawazoe, Y. Site occupation reversal in the Fe-Cr $\sigma$ phase. Phys. Rev. Lett. 1995, 75, 3142-3145.

6. Havránková, J: Vřeštál, J.; Wang, L.G.; Šob, M. Ab initio analysis of energetics of $\sigma$-phase formation in Cr-based systems. Phys. Rev. B 2001, 63, 174104:1-174104:5.

7. Korzhavyi, P.; Sundman, B.; Selleby, M.; Johansson, B. Atomic, electronic, and magnetic structure of iron-based sigma-phases. Mater. Res. Soc. Symp. Proc. 2005, 842, 517-522.

8. Grånäs, O.; Korzhavyi, P.A.; Kissavos, A.E.; Abrikosov, I.A. Theoretical study of the Mo-Ru sigma phase. Calphad 2008, 32, 171-176.

9. Kabliman, E.; Mirzoev, A.; Udovskii, A. First-principles simulation of an ordered sigma phase of the Fe-Cr system in the ferromagnetic state. Phys. Met. Metallogr. 2009, 108, 435-440.

10. Pavlù, J.; Vř̌š́tál, J.; Šob, M. Ab initio study of formation energy and magnetism of sigma phase in $\mathrm{Cr}-\mathrm{Fe}$ and $\mathrm{Cr}-\mathrm{Co}$ systems. Intermetallics 2010, 18, 212-220.

11. Palumbo, M.; Abe, T.; Fries, S.G.; Pasturel, A. First-principles approach to phase stability for a ternary $\sigma$ phase: Application to Cr-Ni-Re. Phys. Rev. B 2011, 83, 144109:1-144109:7.

12. Cieślak, J.; Tobola, J.; Dubiel, S.M. Study of phase stability in the $\sigma$-FeCr system. Intermetallics 2012, 24, 84-88.

13. Cieślak, J.; Tobola, J.; Dubiel, S.M. Formation energy in $\sigma$-phase Fe-V alloys. arXiv:1205.5410v1 [cond-mat.mtrl-sci].

14. Kabliman, E.; Blaha, P.; Schwarz, K.; Ruban, A.V.; Johansson, B. Ab initio-based mean-field theory of the site occupation in the Fe-Cr $\sigma$-phase. Phys. Rev. B 2011, 83, 092201:1-092201:4.

15. Kabliman, E.; Ruban, A.V.; Blaha, P.; Schwarz, K. The atomic site occupancies in the Fe-Cr $\sigma$-phase. Solid. State Phenom. 2011, 170, 13-16.

16. Kabliman, E.; Blaha, P.; Schwarz, K.; Peil, O.E.; Ruban, A.V.; Johansson, B. Configurational thermodynamics of the Fe-Cr $\sigma$-phase. Phys. Rev. B 2011, 84, 184206:1-184206:9.

17. Heine, V.; Joynt, R. Coarse-grained magnetic disorder above $T_{c}$ in iron. Europhys. Lett. 1988, 5, $81-86$.

18. Vitos, L. Total-energy method based on the exact muffin-tin orbitals theory. Phys. Rev. B, 2001, 64, 014107:1-014107:11.

19. Vitos, L.; Abrikosov, I.A.; Johansson, B. Anisotropic lattice distortions in random alloys from first-principles theory. Phys. Rev. Lett. 2001, 87, 156401:1-156401:4.

20. Vitos, L. Computational Quantum Mechanics for Materials Engineers: The EMTO Method and Applications; Springer: London, UK, 2007; p. 247.

21. Perdew, J.P.; Burke, K.; Ernzerhof, M. Generalized gradient approximation made simple. Phys. Rev. Lett. 1996, 77, 3865-3868.

22. Györffy, B. Coherent-potential approximation for a nonoverlapping-muffin-tin-potential model of random substitutional alloys. Phys. Rev. B 1972, 5, 2382-2384.

23. Singh, D.J.; Nordström, L. Planewaves, Pseudopotentials and the LAPW Method, 2nd ed.; Springer: New York, NY, USA, 2006; p. 147.

24. Blaha, P.; Schwarz, K.; Madsen, G.K.H.; Kvasnicka, D.; Luitz, J. WIEN2k, An Augmented Plane Wave + Local Orbitals Program for Calculating Crystal Properties. Karlheinz Schwarz, Technische Universität Wien, Austria, 2001, ISBN 3-9501031-1-2. 
25. Wilson, C.G. Order in binary $\sigma$-phases. Acta Cryst. 1963, 16, 724-730.

26. Sokolovskaya, E.M.; Tuganbaev, M.L.; Stepanova, G.I.; Kazakova, E.F.; Sokolova, I.G. Interaction of cobalt with chromium and rhenium. J. Less-Comm. Met. 1986, 124, L5-L7.

27. Cieślak, J.; Reissner, M.; Dubiel, S.; Costa, B.F.O.; Steiner, W. On the Debye temperature in sigma-phase Fe-V alloys. Intermetallics 2010, 18, 1695-1698.

28. Cieślak, J.; Reissner, M.; Dubiel, S.; Wernish, J.; Steiner, W. Influence of composition and annealing conditions on the site-occupation in the $\sigma$-phase of $\mathrm{Fe}-\mathrm{Cr}$ and $\mathrm{Fe}-\mathrm{V}$ systems. J. Alloy. Compd. 2008, 460, 20-25.

29. Cieślak, J.; Dubiel, S.M.; Reissner, M. Site occupancy and lattice parameters in sigma-phase Co-Cr alloys. Acta Cryst. B 2012, 68, 123-127.

30. Berne, C.; Sluiter, M.; Kawazoe, Y.; Hansen, T.; Pasturel, A. Site occupancy in the Re-W sigma phase. Phys. Rev. B 2001, 64, 144103:1-144103:8.

31. Fries, S.G.; Sundman, B. Using Re-W $\sigma$-phase first-principles results in the Bragg-Williams approximation to calculate finite-temperature thermodynamic properties. Phys. Rev. B 2002, 66, 012203:1-012203:4.

32. Dubiel, S.M.; Cieślak, J. Sigma-phase in Fe-Cr and Fe-V alloy systems and its physical properties. Crit. Rev. Solid State Mater. Sci. 2011, 36, 191-208.

(c) 2012 by the authors; licensee MDPI, Basel, Switzerland. This article is an open access article distributed under the terms and conditions of the Creative Commons Attribution license (http://creativecommons.org/licenses/by/3.0/). 